\title{
Algunos aspectos del desarrollo de la sociedad de servicios
}

Norbis, Mario*

\section{Resumen}

El objetivo de este articulo es presentar algunas de las principales características del cambio de una sociedad industrial a una sociedad de servicios o post-industrial. En una breve reseña histórica este proceso es integrado con el cambio de una sociedad agraria a industrial que tuvo lugar en los Estados Unidos a principios del presente siglo. Las principales características de la sociedad post-industrial son discutidas como también sus implicaciones para la economia de algunos países Latinoamericanos. Los futuros desafíos para la sociedad post-industrial son presentados en forma de preguntas en las conclusiones.

Palabras clave: Sociedad post-industrial, servicios, manufactura, alta tecnología, modelos educativos.

\section{Recibido: 98-06-15 . Aceptado: 98-10-27}

- Ingeniero Químico, Master of Sciences en Ingeniería Industrial e Investigación de Operaciones. PHD. Fue profesor de la Universidad Central de Venezuela, Gerente de operaciones en la industria manufacturera en E.U, miembro del Consejo de la Industria Privada en E.U. Actualmente profesor asociado en el Departamento de Gerencia de la Escuela de Negocios del Quinnipiac College en Handen. Autor de artículos en el área de Gerencia de Operaciones. Autor del libro: Química General Universitaria. E-mail: norbis quinnipiac.edu 


\section{Some Aspects in the Development of the Service Society}

\section{Abstract}

The object of this article is to present some of the most important characteristics observed in the change from an industrial society to a service or post-industrial society. This process is integrated here with previous changes from the agrarian to industrial society that took place in the United States at the beginning of the present century. The main characteristics of the post-industrial society are discussed as well as their implications for some Latin American economies. Future challenges for the post-industrial society are presented in the form of open questions in the conclusions.

Key words: Post-industrial society, services, manufacturing, high technology, educational model.

\section{Introducción}

Los servicios han sido históricamente el centro de la actividad económica, aun en los tiempos en que el principal producto de la economía era agrario o industrial. Entonces, los servicios hacian posible la actividad principal, agraria o industrial, ya fuera mediante el transporte de la materia prima o producto final, el mercadeo y promoción de los productos $o$, en forma más indirecta, proporcionando educación, información o asistencia de salud. Esta interrelación de los servicios con los demás sectores de la economía es presentada gráficamente por Dorothy Riddle (1986: 27) en el Modelo Interactivo de la Economía que reproducimos en el Gráfico 1.

Este articulo se refiere a los servicios, definidos como productos intangibles negociables por sí mismos o conjuntamente con productos manufacturados, como principal producto de la economía y a los cambios, en las sociedades donde se presenta el fenómeno, principalmente Estados Unidos y los países desarrollados.

A comienzos del presente siglo, 3 de cada 10 personas empleadas en los Estados Unidos trabajaban en el área de servicios, el resto en agricultura, minería e industria. En la década de 1950, la mitad del empleo provenfa del sector de servicios. A inicios de la presente década el sector de servicios empleaba 8 de cada 10 trabajadores en los Estados Unidos. El Gráfico 2, con datos y proyecciones de la Oficina de Censos del Departamento de Comercio, (Departamento de Comercio, 1984: 421) muestra la variación del porcentaje de empleo de los tres sectores (Agricultura, Manufactura y Servicios) de la economía en 150 años (1850 - 2000). Por otra parte, la Tabla 1 de Indicadores Económicos (Indicadores Económicos, 1992: 14), muestra la variación en el número de las posiciones de trabajo dispo- 


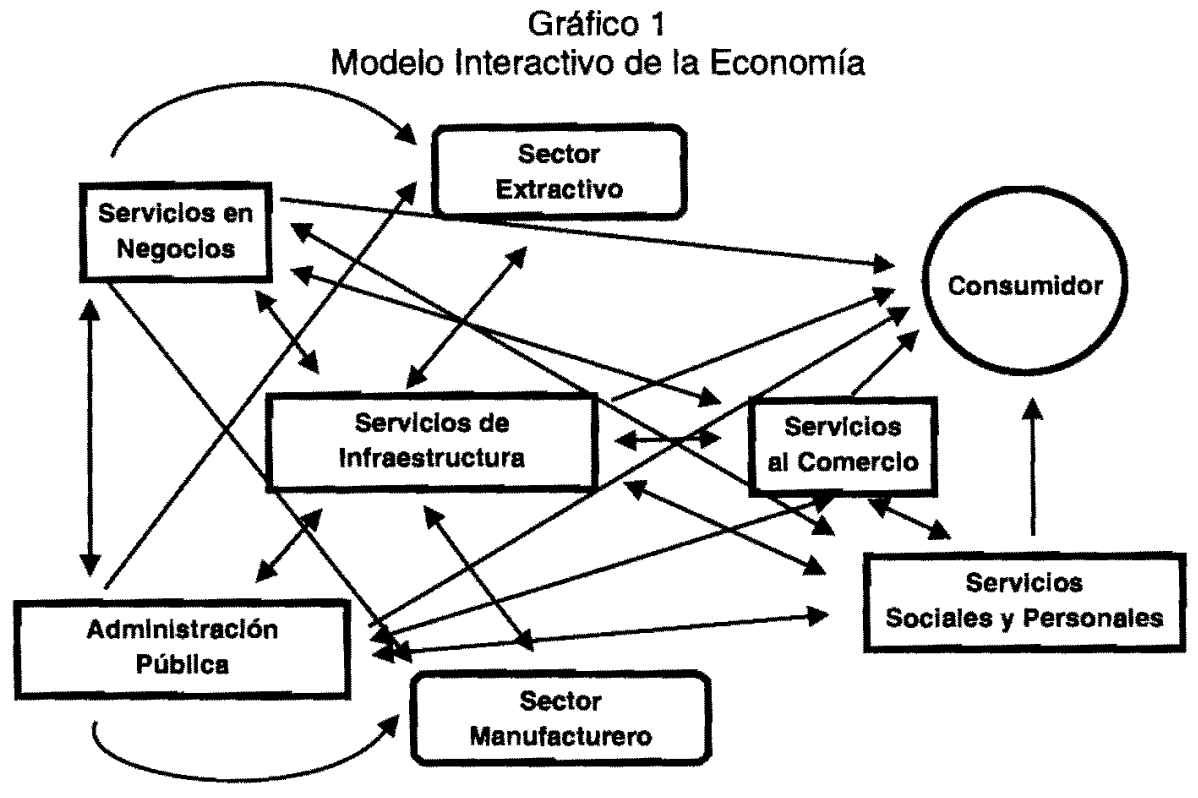

Gráfico 2

Tendencia del Empleo en Estados Unidos por Sectores, 1850 - 1990

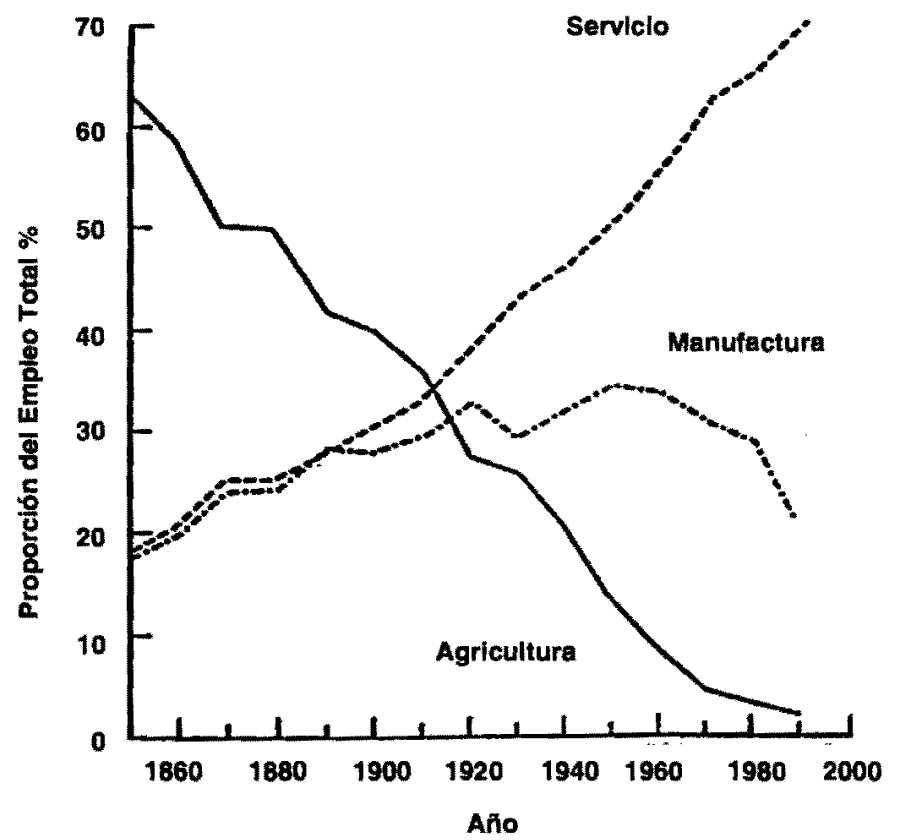


Tabla 1

Velocidad de Aumento de las Oportunidades de Trabajo, por Sectores entre Enero de 1982 y Enero de 1992, en los Estados Unidos, excluyendo Agricultura

\begin{tabular}{|c|c|c|c|c|c|}
\hline & \multicolumn{2}{|c|}{ Enero 1982} & \multicolumn{2}{|c|}{ Enero 1992} & \multirow{2}{*}{$\begin{array}{c}\text { Aumento } \\
\%\end{array}$} \\
\hline & Total (en miles) & $\%$ & Total (en miles) & $\%$ & \\
\hline \multicolumn{6}{|l|}{ Servicios } \\
\hline $\begin{array}{l}\text { Finanzas, Segurose } \\
\text { inmobiliarios }\end{array}$ & 5341 & 6.0 & 6665 & 6.2 & 24.8 \\
\hline Misceláneos & 19036 & 21.3 & 28577 & 26.4 & 50.1 \\
\hline \multicolumn{6}{|l|}{ Gobiemo: } \\
\hline Estatales y Locales & 13098 & 14.6 & 15476 & 14.3 & 18.2 \\
\hline Federal & 2739 & 3.1 & 2981 & 2.8 & 8.8 \\
\hline Ventas Mayor & 5296 & 5.9 & 6010 & 5.6 & 13.5 \\
\hline Ventas Detalle & 15161 & 16.9 & 19118 & 17.7 & 26.1 \\
\hline Transporte & 5082 & 5.7 & 5746 & 5.3 & 13.1 \\
\hline TOTAL & 65753 & 73.5 & 84573 & 78.3 & \\
\hline \multicolumn{6}{|l|}{$\begin{array}{l}\text { Producción de } \\
\text { Blenes: }\end{array}$} \\
\hline Construcción & 3905 & 4.4 & 4587 & 4.2 & 17.5 \\
\hline Minería & 1127 & 1.3 & 657 & 0.6 & -41.7 \\
\hline Manufactura & 18781 & 21.0 & 18283 & 16.9 & -2.7 \\
\hline TOTAL & 23813 & 26.7 & 23527 & 21.7 & \\
\hline GRAN TOTAL & 88566 & & 108100 & 20.7 & \\
\hline
\end{tabular}

nibles entre 1982 y 1992 donde, más allá del aumento del sector de servicios de $73.5 \%$ a $78.3 \%$, destaca la disminución de 500.000 posiciones en el sector manufacturero lo cual es aún más dramático si se considera que la tendencia continuó y se acentuó en los últimos años como se verá más adelante.

Los cambios en el sector ocupacional aquí presentados son la más importante evidencia del avance del sector servicios. En las próximas secciones se analizará las principales razones que condu- jeron a la sociedad de servicios, cuáles son algunas de sus características, qué áreas del sector manufacturero han sobrevivido y aún más, se han expandido, asi como los efectos que estos cambios tienen en otras partes del mundo como por ejemplo América Latina.

\section{Etapas de la economía y el modelo educativo}

La sociedad pre-industrial se caracterizaba por la lucha del hombre por la 
subsistencia, esta lucha era principalmente contra la naturaleza. La actividad humana era una actividad de extracción y la capacidad que el hombre aplicaba predominantemente era su fuerza muscular. Algunas actividades típicas eran la agricultura, la minería, la pesca entre otras. La unidad de vida social y cultural era la familia; el trabajo se organizaba alrededor de la familia, la educación se impartía predominantemente en la familia o grupos de familias. Hoy se podría ver a esta sociedad como una sociedad rutinaria, tradicionalista, basada en autoridad. La mayoría de las economías de Asia y Africa parecen estar todavía en alguna etapa del desarrollo semejante a la descrita. De las economias cuya principal actividad económica deriva de la extracción, sólo algunos países de la Organización de Países Exportadores de Petróleo (OPEP) parecen haber escapado del sub-desarrollo económico.

En la sociedad industrial la actividad dominante es la producción de bienes de consumo, la lucha contra una naturaleza compuesta por ciudades y fábricas se hace empleando energia, que es ahora más accesible, y con máquinas que aumentan la eficiencia del esfuerzo físico. La calidad de la vida se mide por la cantidad de bienes que se posee, el individuo es el centro de actividad social y cultural, respaldado en su trabajo por sindicatos y otras asociaciones gremiales. La economia de la Comunidad Europea y Japón responden todavía a modelos semejantes al descrito.

En la sociedad post-industrial como es llamada por D. Bell (1976: $12-$ 13), la medida del nivel de vida está dada por la calidad de vida en términos de sa- lud, educación, recreación y desarrollo personal.

En esta sociedad la actividad humana es más intelectual y creativa y la vida social se orienta a la comunidad. Las razones para la transición a una sociedad post-industrial son muy variadas; Bell (1976: 124-127), en quien se basa parte de esta sección, sugiere como principales causas el natural desarrollo de los servicios, el aumento de la eficiencia del sector manufacturero que orienta trabajadores a otros sectores y el aumento de la demanda por servicios. La reorientación de los trabajadores hacia diferentes sectores de la economía por el aumento de la productividad en otros sectores es señalada por Clark (1957: 477-480) y hoy es conocida como la hipótesis de ClarkFisher.

Los cambios en la demanda, debidos a la evolución de la sociedad, son presentados como el principal causante de la reorientación de la producción (Johnston, 1988: 202). Así por ejemplo, saturado el mercado de línea blanca del hogar o de televisores y mientras no aparezca una tecnología nueva y revolucionaria en estas áreas (como pudiera ser la televisión interactiva) la producción está destinada a satisfacer al mercado de reposición, o a la reducida demanda de las personas que entran por primera vez al mercado. Por otra parte, los cambios originados en la sociedad asi como la creciente demanda de una más alta calidad de vida en términos de salud, recreación y educación se han traducido en mayor demanda en áreas tales como consultoría, educación y servicios asociados a comunicaciones (especialmente el uso de la red electrónica). Por ejemplo, una hora 
de consulta con un psicoanalista o con un consejero familiar puede resultar hoy más cara que un refrigerador estándar.

Las inversiones gubernamentales, principalmente en educación y salud han sido otro factor de importancia en este proceso, principalmente en los Estados Unidos. La inversión en educación aumentó de 8 mil millones de dólares en 1950 a 151 mil millones en 1979 del los que sólo 25 mil millones es inversión privada. En salud la inversión pasó, en el mismo periodo de tiempo desde $12 \mathrm{mil}$ millones (el estado pagando 1 de cada 5 dólares) a 240 mil millones (el estado pagando 2 de cada 5 dólares) (Ginzberg, 1981: 52).

Por otra parte es posible observar que a medida que decrece el número de empleados en el sector manufacturero en los paises industrializados, aumenta la importación de productos manufacturados de países en desarrollo como lo muestra el Gráfico 3 (El Economista, 1994:3-38).

El sector manufacturero ha logrado desarrollarse y crecer en áreas de alta tecnologia. Este concepto, conocido con el nombre de "High Tech", se refiere a la utilización innovativa de nuevos desarrollos en áreas de electrónica, procesamiento de datos, genética o física de materiales para generar productos o servicios. Estos procesos productivos son en general complejos, de alto riesgo económico y alta rentabilidad (Baridu, 1988: 20-21). A su vez la tecnología, producto de estos procesos, tiene una vida útil muy limitada pues es rápidamente sustituida y mejorada por la competencia o por la misma compañía. Se estima (Baridu, 1988: 20-21) que para el año 2000 la vida media de una tecnología será de seis meses. Bajo estas premisas, operaciones tales como planificación estratégica, planificación de producción, desarrollo tecnológico e implementación, son redefinidas y agrupadas bajo el nombre de planificación de tecnología.

Al mismo tiempo, diferentes modelos educativos han acompañado a estos cambios. El proceso educativo se ha adaptado a diferentes tipos de demanda para producir individuos con aptitud para

\section{Gráfico 3}

Importaciones Manufactureras desde Países en Vías de Desarrollo como Porcentaje del Consumo

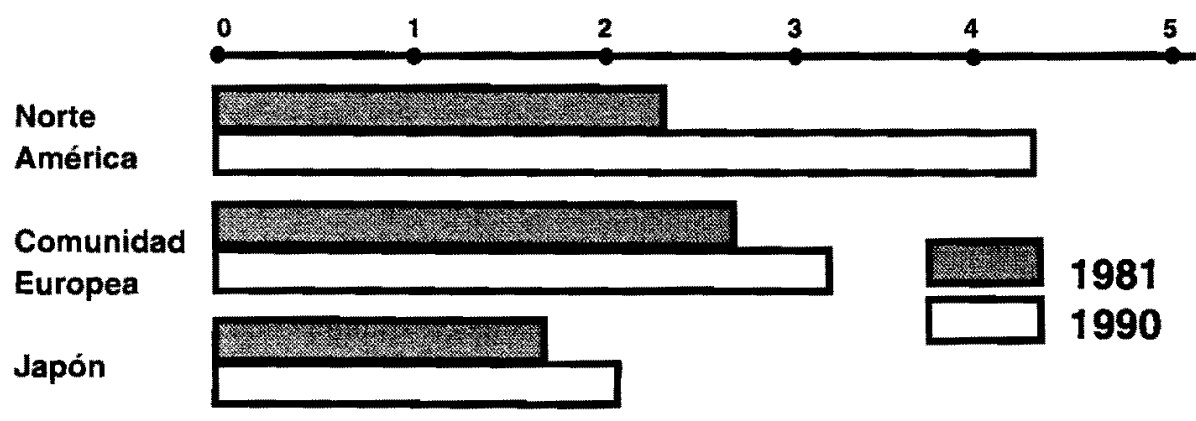


desenvolverse en esa sociedad. Algunas de las caracteristicas más salientes de estos modelos del sistema educativo serán analizados a continuación.

Asociado a cada etapa de la economía existió siempre un modelo educativo. Así, en los tiempos de la economía agraria dominada por la familia, la educación se impartía en la familia o en grupos de pocas familias. Al final de la jornada de trabajo se impartían conocimientos básicos en áreas de lectura, escritura y aritmética para acompañar al entrenamiento práctico recibido durante el día en el campo. La educación/entrenamiento preparaba al educando para sus funciones en la sociedad agraria.

En la sociedad industrial, la educación se organiza en escuelas, con horarios, recreos, uniformes y vacaciones anuales preparando a los educandos para su próxima función en las fábricas.

Los sistemas educativos en general, parecieran haberse tornado demasiado complejos para adaptarse rápidamente a los cambios tecnológicos y sociales. De esta forma, en lugar de ser el motor de los cambios han retrocedido y pueden convertirse en potenciales obstáculos. En particular las escuelas de gerencia están todavía preparando gerentes para la industria manufacturera que muy probablemente terminen administrando empresas de servicios (Johnston, 1988: 209).

El modelo industrial de la educación, que aún existe en la sociedad postindustrial, está dando paso lentamente a un modelo más flexible de educación a distancia con curricula flexibles, parcialmente desarrollados por y para el individuo y sostenido bajo el criterio de la conti- nuidad del proceso educativo en la edad adulta. La educación continua es una de las características más importantes de la educación en la sociedad post-industrial y será motivo de análisis en la próxima sub-sección donde se presentan las principales características de la sociedad post-industrial.

\section{La sociedad de servicios y el papel de la educación}

Algunos de los hechos relativos al advenimiento de la sociedad de servicios o post-industrial y sus características más importantes son:

- En el año 1947 los servicios representaban el $55 \%$ del Producto Bruto Nacional de los Estados Unidos, en 1975 pasaron a representar el $66 \%$ (Departamento de Comercio, 1975: 137).

- En el año 1956 el número de trabajadores de "cuello blanco" pasó al número de trabajadores de "cuello azul", ver distribución histórica del empleo en el Gráfico 4 (Departamento de Comercio, 1975: 137).

- El conocimiento teórico y aplicado es la fuerza dominante y la investigación es la principal actividad asociada con él. Las industrias líderes se caracterizan por sus actividades de investigación, ésto se puede ver comparando por ejemplo industrias líderes de hoy (IBM, Industrias en Ingenieria Genética) y su dependencia de la investigación con la de industrias líderes de los años 1960's (General Motors) o de los años 1950's (American Steel). Asimismo, la inversión del gobierno de los Estados Unidos en investiga- 


\section{Gráfico 4}

Distribución del Empleo en los Estados Unidos. 1900-1990

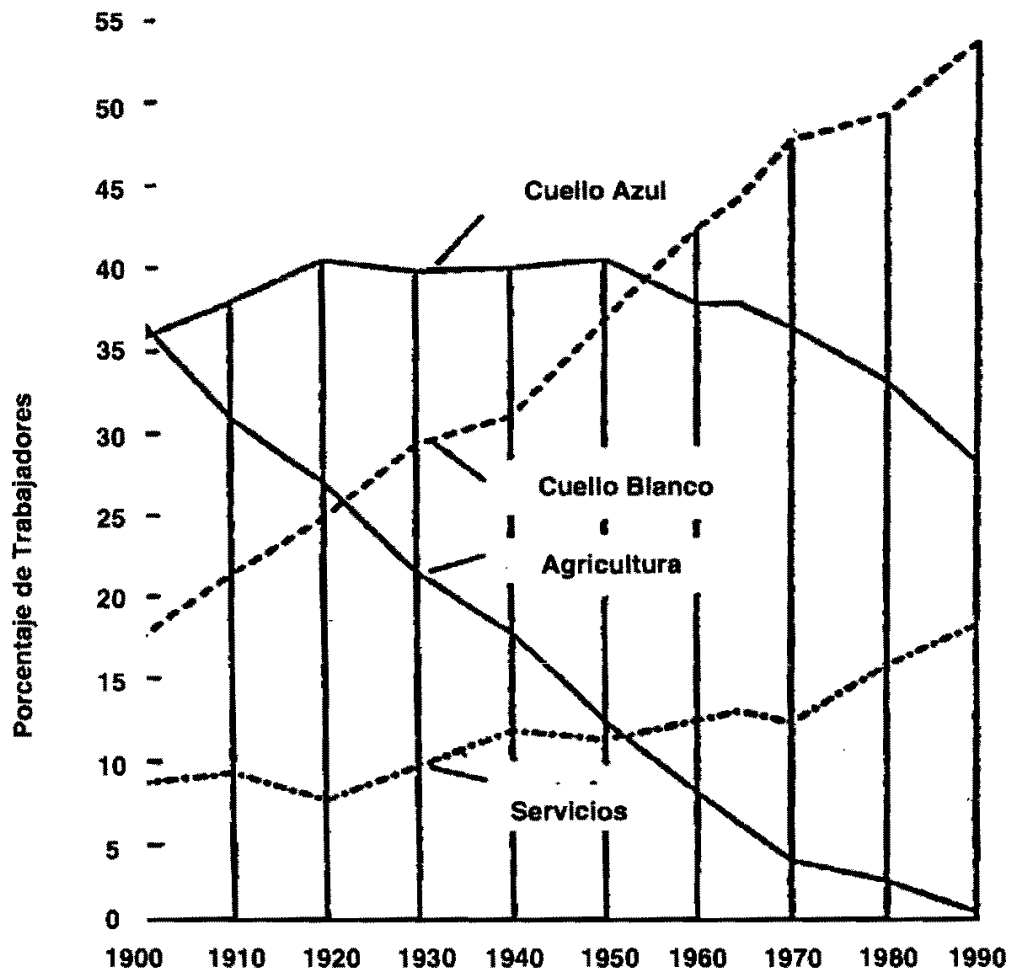

Año

ción y desarrollo pasó de 1000 millones de dólares en 1965 a $12.000 \mathrm{mi}$ llones de dólares en 1995 (ver Gráfico 5) (Fundación Nacional para la Ciencia, NSF, 1997).

- La planificación que acompaña al desarrollo tecnológico, conocida como Planificación Tecnológica (Baridu, 1988: 22-23), ha estado principalmente enfocada a la anticipación y neutralización de efectos laterales, por ejemplo, cuando la industria bancaria introduce Transferencia Electrónica de
Fondos, la flexibilidad del cliente entre que un cheque es pagado y el mismo es cobrado, conocida como "float" se pierde y por ello nuevas medidas se hacen necesarias para mejorar los servicios a los usuarios.

- Una nueva clase intelectual/tecnológica se desarrolla cuya principal posesión es su educación y cuyo principal desafío es la eficiente administración de sistemas cada vez más complejos (Bell, 1976: 15-20). 


\section{Gráfico 5}

Inversión del Gobierno Federal de los Estados Unidos en Investigación

y Desarrollo 1963-1995 (en dólares constantes 1984)

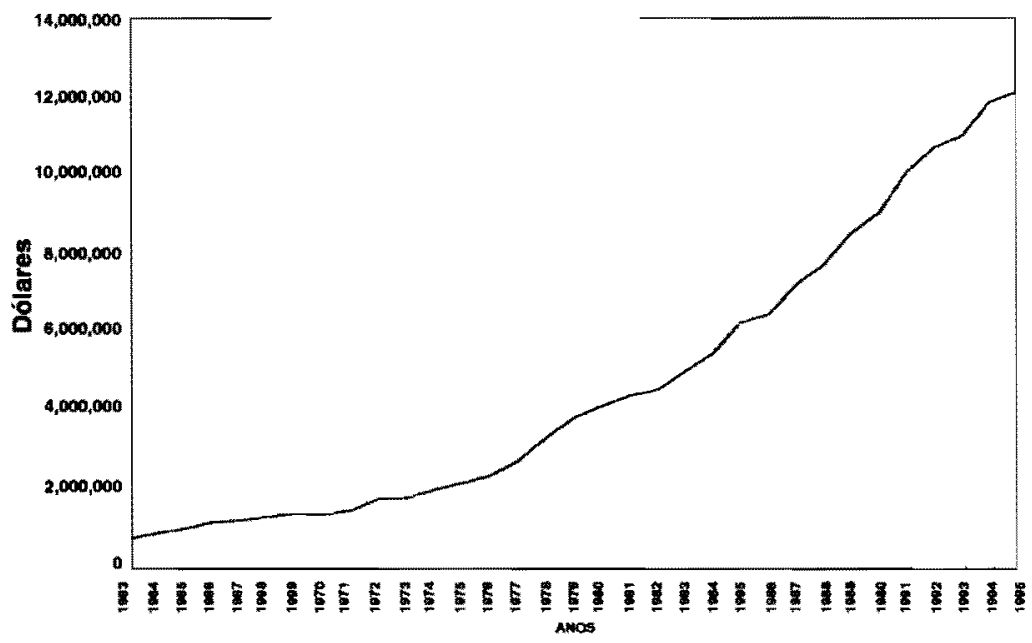

- Los continuos cambios en las habilidades que el mercado demanda de los individuos, desarrollan en ellos la necesidad de continuar su proceso educativo en la edad adulta, prácticamente durante toda su vida productiva. En respuesta a esta necesidad las empresas desarrollan procesos internos de entrenamiento y reentrenamiento de los mismos individuos en diferentes áreas para poder conservarlo en el proceso productivo y al mismo tiempo aumentar su productividad.

Las universidades responden a esta necesidad desarrollando programas de Educación Continua destinados a proveer de más flexibilidad a los profesionales graduados. En los Estados Unidos y en Venezuela, por ejemplo, en las décadas de 1980 y 1990 se vuelven populares programas de maestría en gerencia de salud y en gerencia de empresa para reentrenar a médicos e ingenieros que, sin un entrenamiento formal en gerencia administrativa han llegado a las posiciones gerenciales de la empresa.

Finalmente el tercer componente del triángulo de Educación Continua es el Estado el cual desarrolla y financia programas destinados a reentrenar a trabajadores "dislocados", definidos como aquellos trabajadores con habilidades cuya demanda ha disminuido (operaciones de manufactura básicas como soldadura o tornería por ejemplos) y para los cuales es más económico (y humano) pagarles su nuevo entrenamiento que la compensación social por desempleo. Aun más, los programas estatales tratan de anticipar situaciones de despido/dislocación financiando directamente a la em- 


\section{Gráfico 6}

\section{Inversión Total del Estado de Massachusetts en el Desarrollo} de su Fuerza Laboral (Mass. Job Counsil, 1993)

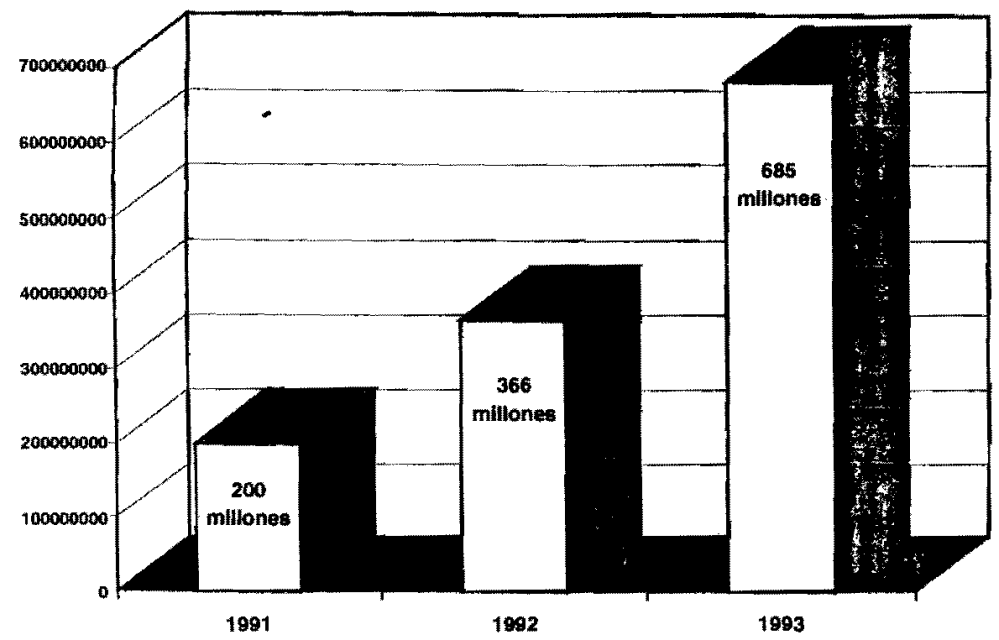

presa para que ella provea el reentrenamiento in situ, estos programas se llaman "On the Job Training" (OJT) o "Entrenamiento en el Trabajo".

El Gráfico 6 muestra en forma comparativa la inversión total que el Estado de Massachusetts $(27000 \mathrm{Km} 2,6$ millones de habitantes, 3.5 millones de personas aptas para el trabajo y $5 \%$ de desempleados) (Boletín Económico de Massachusetts, 1995), ha hecho para el desarrollo y promoción de la fuerza laboral del estado en los años fiscales 1991, 1992 y 1993. Sobresale, más allá de la magnitud de la inversión, su crecimiento al multiplicarse por 3.4 en dos años.

Finalmente, como otras características menores pero detectables de la sociedad Post-Industrial, pueden mencionarse tiempo flexible para el trabajo, trabajo desde la casa y servicios incorporados, en los que la empresa ofrece desde de atención diaria de infantes hasta atención psicológica, pasando por senvicios de viajes y seguros.

\section{Implicaciones para la economia latinoamericana}

El desarrollo de una economía de servicios en Estados Unidos pareciera haber motivado una mayor reorientación del sector productivo en los países en desarrollo hacia áreas de manufactura que, por haber sido descontinuadas en los Estados Unidos son ahora importadas por el mercado norteamericano. $O$ ¿fue quizás que la baja del precio (y rentabilidad) de los productos manufacturados por la entrada de otros proveedores en el mercado, empujó a la economía norteamericana a un sector de servicios de aparente mayor rentabilidad? (Johnston, 1988: 202-203) 
Independientemente de qué fenómeno fue causa y cuál fue efecto, es evidente que aquellas economías latinoamericanas más influenciadas por la economia estadounidense (México, Brasil) están desarrollando su planta manufacturera para proveer al mercado norteamericano. Estados Unidos y Canadá están dando los pasos necesarios para fluidificar este proceso a través de la legislación NAFTA (North Atlantic Free Trade Agreement) y algunos países latinoamericanos adecúan su legislación para estimular la exportación manufacturera (Johnson, 1995: 178-182).

Desde este punto de vista, un gran reto para las economías latinoamericanas consiste en utilizar esta bonanza temporal para desarrollar una sociedad, que por tener un más alto nivel de vida, genere una demanda de servicios que produzca el embrión de una economía de servicios local. Otro reto consiste en invertir en la educación/entrenamiento de sector productivo para que el mismo se pueda orientar hacia proyectos de mayor demanda tecnológica que, en el largo plazo remunerarán mejor el esfuerzo.

\section{Algunas conclusiones}

Los cambios detectados en el sector de empleos que acompañan a la transformación de la sociedad industrial son presentados como introducción a este trabajo. Estos cambios son analizados como una nueva etapa en el desarrollo de la sociedad que había ya evolucionado de agraria a industrial. El avance cualitativo de la demanda es presentado como uno de los factores más importantes de este proceso. La evolución de los modelos educativos en este proceso y el hecho de que ellos parezcan ir a la saga del proceso en lugar de ser motivantes del proceso, también es discutido junto a las caracteristicas más importantes de la economía post-industrial. Son discutidos que estos cambios pueden tener en el área de mayor influencia económica de los Estados Unidos.

La economía de servicios o economía post-industrial se presenta como un nuevo y permanente estado del desarrollo económico, como lo fueron antes la economía agraria y la economía industrial, y los Estados Unidos aparecen como la primera economia post-industrial del mundo. Otras economías se irian integrando a la vez que se vuelvan hacia los servicios y manufactura de alta tecnología. Simultáneamente otras economías es posible que emerjan como lideres manufactureros entre ellas las siete naciones del sudeste asiático, Chile y México en América Latina y, China como lo pronostica El Economista (EI Economista, 1994:3-38).

Junto con el desarrollo de la econo. mía post-industrial, se presenta la necesidad de nuevos procedimientos para evaluar productividad (National Research Council, 1994: 44-45), controlar inventarios y pronosticar demandas en servicios, en otras palabras "nuevas modalidades de producción" (Hirschhorn, 1988: 22). Estas eran operaciones regulares de la industria manufacturera, ¿es válido transplantar metodologías y adaptarlas a servicios? o ¿es necesario desarrollar metodologías completamente nuevas para las operaciones en servicios? Hasta ahora estas metodologias han sido transplantadas y ligeramente adaptadas a la 
industria de servicios. El desafio consiste en el desarrollo de nuevas metodologías de operaciones especificas para el área de servicios y de "alta tecnología", principales componentes de la economla post-industrial.

Por otra parte cuando el sector de servicios desarrolle algunas estrategias propias especialmente en áreas de mercadeo y calidad (Heskett, 1987: 118-120) bien pudieran ser utilizadas para mejorar la productividad en áreas de producción manufacturera.

En el área de gerencia, se podría anticipar que gerenciar en servicios implicará procedimientos de alguna manera diferentes de aquellos utilizados para gerenciar empresas manufactureras (Fritzsimmons, 1994: 12-16) representando un nuevo desafío para el sistema educativo que deberá formar gerentes con la capacidad y la visión para innovar procedimientos que contribuyan al continuo mejoramiento de la calidad, la productividad $y$, finalmente, las condiciones de vida.

Finalmente, cabe preguntarse: ¿es factible una economía basada casi exclusivamente en servicios? Para responder a esta pregunta recordemos que vivimos épocas de cambio, y ciertamente a comienzos de este siglo, una sociedad con menos información que la actual, vería con grandes dudas su futura subsistencia ante el abandono de una economía agraria por una parte mayoritaria de la población que emigraba a las ciudades para in. corporarse a la economia industrial. Esa sociedad se preguntaría entonces si sería factible una economía basada casi exclusivamente en la industria manufacturera...

\section{Bibliografía citada}

BARIDU, Adedeji (1988), Project Management in Manufacturing and High Technology Operations, J. Wiley \& Son, New York.

BELL, Daniel (1976), The Comming of PostIndustrial Society: A Venture in Social Forecasting. Basic Books Inc., New York.

BOLETIN ECONOMICO DE MASSACHUSETTS (1995), Departamento de Empleo y Entrenamiento, Julio 1995.

CLARK, Colin (1957), The Conditions of Economic Progress, The Macmillan Co. London.

DEPARTAMENTO DE COMERCIO (1975), Oficina de Censo, Estadísticas Históricas de los Estados Unidos, Pág. 137.

DEPARTAMENTO DE COMERCIO (1984), Oficina de Censo, Resúmenes Estadísticos de los Estados Unidos, Pág. 421.

EL ECONOMISTA (1994), War of the Worlds, Vol. 333, No. 7883, Pág. 3-38, Octubre 1994.

FRITZSIMMONS, James, FRITZSIMMONS, Mona, (1994) Service Management for Competitive Advantage, McGraw-Hill, Inc. New York.

GINZBERG, Eli, VOJTA, George (1981), The Service Sector of the U.S. Economy, Scientific America, Vol. 244 , No. 3 , Pág. 48-55, Marzo 1981.

HESKETT, James (1987) Lessons in the Service Sector, Harvard Business Review, Pág. 118-126, Marzo-Abril 1987.

HIRSCHHORN, Larry (1988) The PostIndustrial Economy: Labour, Skills and the New Mode of Production, The Service Industries Journal, Vol. 8 , No. 1, Pág. 19-38. 
INDICADORES ECONOMICOS (1992), Reporte preparado por el Consejo de Asesores Económicos para el Comité Económicos Conjunto, Oficina de imprenta del Gobiemo de los Estados Unidos, Pág. 14, Junio 1992.

JOHNSON, Hazel (1995) Banking Without Borders, Challenges and Opportunities in the era of NAFTA and the Emerging Global Marketplace, Probus Publishing, Chicago.

JOHNSTON, Robert (1988), Service Industries: Improving Competitive Performance, The Service Industries Journal, Vol. 8, No. 2, Pág. 202-211.
NATIONAL SCIENCE FOUNDATION (1995), Survey of Federal Science and Engineering Support to Universities, Colleges and Nonprofit Institutions. Año Fiscal 1995. Tabla B-1.

NATIONAL RESEARCH COUNCIL (1994) Information Technology in the Service Society, A Twenty First Century Level, Computer Science and Telecomunication Board, National Academic Press, Washington D.C.

RIDDLE, Dorothy (1986), Service-Led Growth, Praeger Pub., New York. 